\title{
Preliminary Sketch of Medical Residency in Cardiology. Committee of Medical Residency and Training in Cardiology SBC/FUNCOR
}

\author{
Bráulio Luna $\mathrm{F}^{\circ}$, Valter C. Lima, Celso Ferreira $\mathrm{F}^{\circ}$, Carlos Gun, Orlando Campos $\mathrm{F}^{\circ}$, \\ Rui Póvoa, Ângelo A. V. de Paola, Celso Ferreira
}

São Paulo, SP - Brazil

In mid-1997, the new board of directors of SBC/ FUNCOR established the Committee of Medical Residency and Training to assist with the evaluation of training in cardiology. Besides providing subsidies and criteria for accreditation of the courses, this committee was encouraged to address the problem of cardiologists' training in our country. During six months of work, the committee concluded that, despite the apparent dichotomy between the official programs of Medical Residency (MR) approved by the Brazilian Department of Education and Culture and those endorsed by SBC/FUNCOR, the establishment of a link between the two institutions was a priority. The following proposal resulted from this recognition of the need for greater cooperation and was developed from contributions of participants from the main cardiology MR program in São Paulo state, during the seminar "Medical Residency and Brazilian Society of Cardiology", held on February 7, 1998 at the SBC/FUNCOR headquarters. Subsequently with the approval of the board of directors of SBC, this project was presented to the National Committee of Medical Residency (NCMR) in April 1998, and was well received by its members. Both groups recognized that a proposal was needed. In conjunction with NCMR, a national seminar would be held to carefully examine and define the main consensual proposals.

We present here the proposal in its preliminary form in the hopes of inspiring discussion of this significant topic affecting the evolution of cardiological education in our country.

\section{Introduction and historical summary}

The Medical Residency is the best form of training and specialization in the medical arena. It has been in existence in Brazil since the 1940s. Initially it was linked to university hospitals but, subsequently, it expanded to other public and private non-academic institutions. Its broad acceptance by health institutions and economical and market forces

Mailing adress: Bráulio Luna $\mathrm{F}^{\circ}-\mathrm{SBC} / \mathrm{FUNCOR}$ - Rua Beira Rio, 45 - $3^{\circ}$ 04548-050 - São Paulo, SP - Brazil embracing medical specialists, and sophisticated technologies, as well as the pedagogical deficiencies in medical courses, caused a distortion of the conceptions that originally called for the establishment of medical residency training.

Subsequently dozens of medical residency programs arose based on distortions of the original concepts, using recently graduated physicians as cheap labor, adding additional disgrace to the health care labor market. This fact gave rise to accusations that MR was a privileged instrument for exploitation of qualified labor. Therefore, residents made many demands to improve working conditions and fairness in the health care labor market. This resulted in the approval of law 80,281 by the federal government in 1977. It defined the meaning of medical residency and created the NCMR. Afterwards, law 6,932/81 was enacted. It governed the activities of medical residents.

Currently, there are also the State Committees of Medical Residency (SCMR) that, along with NCMR, are responsible for the accreditation and supervision of medical residency programs. Within this legal framework, the officially accredited programs provide certificates of specialization recognized in all areas of the labor market. Alternatively, one can obtain a specialist title by undergoing examinations comprising tests and title evaluations performed by the societies of medical specialties with the consent of the Brazilian Medical Association and acknowledgement of the Federal Board of Medicine (Partnership agreement AMB/CFM/1988).

This duality of criteria for obtaining a specialist title has complex ramifications for medical practice. In addition to the variations in the quality of the different MR programs, inspection and control by NCMR and SCMR have not guaranteed an acceptable quality. In addition, two hypothetical types of specialists have been created - one who has completed MR and therefore holds the title issued by NCMR and the other who has received his/her title from the societies of specialists, as already explained. It is believed that the participation of the societies of specialists in this process would be of greatest importance in establishing uniformity and minimum professional qualifications in the service of society. 
General profile of the cardiologist - The specialist must have broad training in internal medicine, which enables him/her to practice in the different areas of cardiology. This training takes for granted a close and logical relationship among the different organs and systems of the human body, emphasizing the interaction with the cardiovascular system both in its general and specific aspects.

Minimum competence - Our approach will be divided in two levels. The first level is related to the objectives and the second to the contents of the program.

In regard to the objectives, we will consider the following: What is expected from the specialist and how the institution can participate in his/her medical education?

In regard to the contents of the program, we will focus on the following: a) What is considered basic for the training of a cardiologist?; b) how to acquire critical and constructive knowledge for professional practice?; c) what should be the minimum length of time spent in training in the different sectors, for example, the training period in the ward, in the outpatient care unit, etc.

To facilitate the analysis of the questions about minimum competence, we will take two approaches, the institutional and the programmatic one.

Institutional level - The services where medical residency programs will be carried out should have a registration unit, an emergency unit, an intensive care unit, an outpatient care unit, and units for invasive and noninvasive diagnostic methods. It is also advisable to include a training program with interface between cardiac surgery and clinical cardiology.

The institutions should have libraries available for medical residents provided with basic texts on cardiology and internal medicine and the main periodicals of the specialty, as well.

Programmatic level -a) Pre-requisite: A two-year training in internal medicine in a program of medical residency acknowledged by the NCMR; b) duration - A minimum of 24 months, but 36 months being the recommended optimal period; c) contents - during the training, the cardiologist should develop cognitive and practical skills that enable him/her not only to recognize and treat the different heart diseases but also to acquire critical reasoning in the utilization of different established or experimental management techniques in cardiology. This will be accomplished through classes, seminars, scientific meetings, visits to ward, discussions of manuscripts, etc.

According to what was explained, the following topics should be considered: Basic topics: Cardiac metabolism, physiology, primer of molecular biology in heart diseases, electrophysiology, neural and humoral control of the cardiovascular system, lectures of clinical epidemiology and scientific methodology; practical topics: Cardiac insufficiency, acute and chronic coronary insufficiency, congenital heart diseases, valvular heart disease and rheumatic diseases, cardiomyopathies and Chagas' disease, dyslipidemia, hypertension, ECG, arrhythmia, hemodynamics, and correlated diagnostic methods; d) hands on training - the training must be developed through clinical and diagnostic, anatomico-clinical, and clinical-surgical meetings, and rotatory trainings distributed as follows: Registration unit: medical assistance to patients admitted to the wards, emergency department, intensive care unit, coronary care unit, and consultations with specialists of other areas. These activities should take up to $60 \%$ of the total training time; outpatient Care Unit: $20 \%$ training time should be spent in outpatient care; diagnostic methods: active participation in invasive and non-invasive procedures is advisable and should represent $20 \%$ of the training time; e) teaching method - A cardiologist with an academic title or one acknowledged by SBC/BMA should always supervise the program. In addition to the usual pedagogical methods, the teaching of basic techniques of clinical research and statistics should be a central part of the training, aiming to provide the medical resident with a critical spirit and intellectual initiative; f) evaluation - institutional - The institution can establish its own criteria that should be very clear and unambiguous. Besides the traditional subjective evaluation during work, there should also be objective ones, such as written or practical examinations; extra-institutionalSBC/FUNCOR will annually apply specific tests developed by specialists related to the topics covered and the skills progressively acquired during the training of medical residents, for first-, second-, and third-year trainees. These tests will be used to evaluate the medical residents and the services as well, being one of the criteria for reaccreditation.

At the end of the program, the cardiologist, in addition to recognizing and managing cardiac pathologies, should be able to: 1) Prescribe and interpret cardiological examinations, ECG, chest roentgenograms, hemodynamic, cineangiocardiographic and radioisotopic examinations, and cardiac biopsy; 2) handle drugs and apply adequate therapeutical methods; 3) identify patients for heart transplantation, for surgical treatment and for angioplastic and valvoplastic treatment; 4) recognize and treat the psychosomatic aspects related to cardiac patients; 5) allow the continuing interest in clinical investigation, stimulating the criticalscientific spirit and utilization and adequate incorporation of medical advances in cardiology.

\section{Conclusion}

This preliminary sketch represents a contribution to the debate about the enhancement of the cardiology MR programs in Brazil. It does not pretend to be original, because it was based on other proposals. It does not pretend to be a finished project as it only proposes to initiate the discussion about the quality of the MR and the role of SBC/ FUNCOR in its improvement.

To guarantee that the cardiology MR programs are of the highest quality and are adequate to the needs of Brazilian society, SBC/FUNCOR should develop a strategy and 
efforts that consider the already existing setting and enable a partnership with the agencies responsible for the MR in this country.

Therefore, it is considered fundamental that the ongoing programs, as well as the ones that will be established, also have the approval of SBC/FUNCOR. Currently, this function, due to the legal framework of the country, is the responsibility of the NCMR and SCMR. Although historically this designation has represented an advance in the evolution of MR in Brazil, it is known that this form of accreditation and evaluation of MR has deficiencies inherent in the enormity and complexity of the programs of MR spread throughout a country with continental dimensions.
Thus, the participation of SBC/FUNCOR in the initial analysis of the programs in the accreditation phase would be a natural solution, as well as in the continuing evaluation of dozens of ongoing programs in the country. Considering the structure of SBC/FUNCOR with societies of specialists in all Brazilian states, added to the natural easiness of specialities evaluating related programs, we could act as highly qualified selectors to NCMR and SCMRs. At the same way, the reevaluations of the different programs for reaccreditation would be much easier and more natural.

Very soon, in a symposium especially devoted to discussing cardiology medical residency in Brazil, we hope to deepen the discussion of this and other proposals.

\section{References}

x. American College of Cardiology - COCATS Guidelines. Guideline for training in adult cardiovascular medicine. Core Cardiology Training Symposium. June 27-28, 1994. J Am Coll Cardiol 1995, 25: 1-34.

x. Fundação do Desenvolvimento Administrativo (FUNDAP) - Requisitos Mínimos de um Programa de Residência Médica: Competências em Cardiologia. Documentos de Trabalho 1991; 50: 6-32. x. Sampaio SAP - A implantação da residência médica no Hospital das Clínicas: 40 anos de história. ESTUDOS FUNDAP - Residência Médica 1984; 4-32.

x. Residência Médica em Cardiologia - Escola Paulista de Medicina. Departamento de Medicina, UNIFESP-EPM, 1997.

x. Centro de Estudos e Coordenação de Bolsas e Estágios (CECBE) - FUNDAP A Residência Médica no Estado de São Paulo: Conceitos e Análises. Documentos de Trabalho 1987; 4-94. 